\section{BRAZIULIAN JOURNAL \\ OF MIEDICAL AND BIOLOGICAL RESFARCH}

www.bjournal.com.br
ISSN 0100-879X

Volume 42 (8) 692-775 August 2009

CLINICAL INVESTIGATION

Braz J Med Biol Res, August 2009, Volume 42(8) 712-716

Classification and hearing evolution of patients with sudden sensorineural hearing loss

N.O. Penido, O.L.M. Cruz, A. Zanoni and D.P. Inoue

The Brazilian Journal of Medical and Biological Research is partially financed by
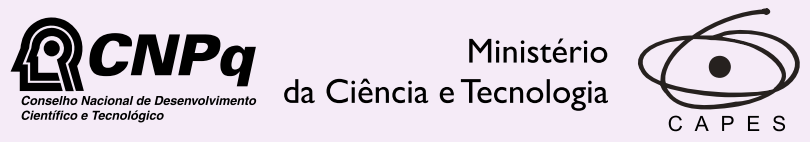

Ministério da Educação
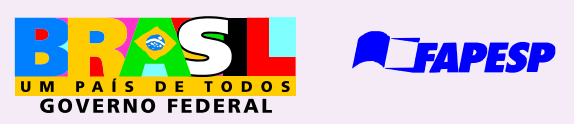

Institutional Sponsors 


\title{
Classification and hearing evolution of patients with sudden sensorineural hearing loss
}

\author{
N.O. Penido, O.L.M. Cruz, A. Zanoni and D.P. Inoue \\ Departamento de Otorrinolaringologia e Cirurgia de Cabeça e Pescoço, Escola Paulista de Medicina, \\ Universidade Federal de São Paulo, São Paulo, SP, Brasil \\ Correspondence to: N.O. Penido, Rua René Zamlutti, 160/131, 04116-260 São Paulo, SP, Brasil \\ Fax: +55-11-3141-9878. E-mail: nopenido@terra.com.br
}

\begin{abstract}
The aim of this study was to analyze clinical aspects, hearing evolution and efficacy of clinical treatment of patients with sudden sensorineural hearing loss (SSNHL). This was a prospective clinical study of 136 consecutive patients with SSNHL divided into three groups after diagnostic evaluation: patients with defined etiology (DE, $N=13,10 \%)$, concurrent diseases $(C D, N=63$, 46.04\%) and idiopathic sudden sensorineural hearing loss (ISSHL, N =60,43.9\%). Initial treatment consisted of prednisone and pentoxifylline. Clinical aspects and hearing evolution for up to 6 months were evaluated. Group CD comprised $73 \%$ of patients with metabolic decompensation in the initial evaluation and was significantly older (53.80 years) than groups DE (41.93 years) and ISSHL (39.13 years). Comparison of the mean initial and final hearing loss of the three groups revealed a significant hearing improvement for group CD $(P=0.001)$ and group ISSHL $(P=0.001)$. Group DE did not present a significant difference in thresholds. The clinical classification for SSNHL allows the identification of significant differences regarding age, initial and final hearing impairment and likelihood of response to therapy. Elevated age and presence of coexisting disease were associated with a greater initial hearing impact and poorer hearing recovery after 6 months. Patients with defined etiology presented a much more limited response to therapy. The occurrence of decompensated metabolic and cardiovascular diseases and the possibility of first manifestation of auto-immune disease and cerebello-pontine angle tumors justify an adequate protocol for investigation of SSNHL.
\end{abstract}

Key words: Sensorineural hearing loss; Sudden deafness; Prognosis

Received August 22, 2008. Accepted May 8, 2009

\section{Introduction}

The term sudden deafness is frequently used in the scientific literature; however, its definition is neither precise nor completely adequate to describe sudden sensorineural hearing loss (SSNHL), which is still commonly confused with idiopathic sudden deafness. Furthermore, there is no universally accepted definition of what constitutes SSNHL, either in terms of the amount of hearing loss or the time interval during which it must develop. To compare literature data and analyze our cases, we have chosen the most frequently used definition of sudden deafness: the loss of hearing of at least $30 \mathrm{~dB}$ over at least 3 contiguous test frequencies occurring within 3 days $(1,2)$. It is important to emphasize that this is a definition of one symptom and, since 1944 when De Kleyn (3) first reported
SSNHL, the scientific community is still searching for answers regarding the clinical aspects, treatment and evolution of SSNHL. As a symptom, SSNHL can be found in several diseases such as temporal bone or cerebellopontine angle tumors, inflammatory inner ear disorders and Ménière's disease (4).

Usually, no etiology can be identified after clinical evaluation, even after using a significant number of diagnostic tools, including magnetic resonance imaging (MRI) (5). Under these circumstances, after an adequate etiological search, the hearing loss can be termed idiopathic. However, the low specificity and high cost of the complementary exams currently available do not stimulate the interest in establishing an etiologic diagnosis. The identification of cases with specific and treatable causes is critical to undertaking optimum management and obtaining the 
most favorable outcome.

SSNHL can also occur in patients presenting concurrent diseases such as hypertension, diabetes and dyslipidemia where a causal relation with cochlear damage is uncertain but probable (6).

In this prospective study, we investigated the clinical aspects, hearing evolution and possible efficacy of clinical treatment in three different groups of patients with SSNHL: patients with defined etiology, patients with concurrent diseases and patients with unknown etiology (idiopathic).

\section{Patients and Methods}

One hundred and thirty-six consecutive patients (139 ears) who developed hearing losses of at least $30 \mathrm{~dB}$ in three contiguous frequencies during a period of no more than 3 days were evaluated in our Department between March 2000 and December 2006. All patients were seen within 20 days of the onset of hearing loss and none received prior treatment.

All patients underwent a standard evaluation consisting of a detailed medical history and physical examination, audiometric testing, blood tests, and imaging study.

The Federal University of São Paulo Ethics Committee approved the research (\#0316/08) and written informed consent was given by the patients.

Audiometric tests consisted of pure-tone audiometry, speech audiometry (speech reception threshold test and speech discrimination score), and tympanometry curve with determination of acoustic reflexes. The hearing involvement was monitored by successive audiometric examinations: on the day of presentation, weekly during the 1st month, and at 3 and 6 months after presentation. A pure tone average of $500,1000,2000$, and $4000 \mathrm{~Hz}$ was used to categorize the losses as mild (26-40 dB), moderate (41-70 $d B$ ), severe (71-90 dB), profound (>90 dB), or total.

$M R I$ images of the encephalon with emphasis on the temporal bone and posterior fossa were obtained in a sequence concentrated on T1 Turbo Spin Echo (TSE) on axial and coronal planes, with and without contrast, in 1.0$\mathrm{mm}$ thick sections. T2 images on the coronal plane in TSE and 3-D using $0.7-\mathrm{mm}$ reconstructions were also collected. The exam was completed with axial sections in a concentrated sequence in fluid attenuation inversion recovery to assess the brain.

Initial laboratory tests included complete blood count, determination of fasting glucose, total and fractionated cholesterol and triglycerides, and erythrocyte sedimentation rate. All exams were performed before or on the first day of treatment.

During follow-up, some complementary investigations such as distortion product otoacoustic emissions, electrocochleography, laboratory tests for HSP 70 protein (68 $\mathrm{kD}$ ), rheumatologic tests (anti-nucleus factor), determination of complement and viral serology (mumps, herpes simplex types I and II, varicella zoster, cytomegalovirus, HIV, and mononucleosis) were carried out in cases of worsening of hearing thresholds or changes in patient clinical condition, or based on the results of MRI.

The diagnosis of Ménière disease followed the criteria defined by the Committee on Hearing and Equilibrium of the American Academy of Otolaryngology - Head and Neck Surgery Foundation (7).

The patients were divided into three groups: SSNHL with defined etiology (DE), SSNHL with concurrent disease (CD), and idiopathic sudden sensorineural hearing loss (ISSHL) according to the results of clinical, imaging, and biochemical evaluations.

All subjects received prednisone and pentoxifylline after the first evaluation. The initial dosage of prednisone was $1 \mathrm{mg} / \mathrm{kg}$ (60 mg/day maximum) by mouth daily for 5 days, then tapered-off and discontinued after 15 days. Oral pentoxifylline at the dose of $1200 \mathrm{mg} /$ day divided into three doses was initiated together with prednisone and continued for a minimum of 4 and a maximum of 8 weeks. All patients were treated as outpatients. Patients who required specific therapy for diabetes, hypertension and dyslipidemia were treated in association with the proposed standard therapy. No surgical intervention was performed during the 6-month period of follow-up.

The percentage of hearing recovery was calculated using the following measurement: recovery rate $(\%)=$ (initial mean threshold - final mean threshold) x 100 / (initial mean threshold - 25).

Using $25 \mathrm{~dB}$ (hearing level) as normal hearing threshold allows a universal use of this form of hearing restoration measurement, even in cases with bilateral involvement when the use of contralateral side as reference is not possible (8). The mean arithmetical initial and final hearing threshold was calculated based on thresholds of $500,1000,2000$, and $4000 \mathrm{~Hz}$. Recovery rates over $90 \%$ were considered total recovery and values between 21 and $90 \%$ were considered as partial recovery. Values below $20 \%$ represented lack of recovery, whereas negative values denoted worsening of hearing thresholds (4). To assess the supposed effect of treatment and hearing evolution we compared the initial and final hearing thresholds at 6 months in the three groups using the ANOVA statistical method. The Pearson $\chi^{2}$ test was employed to assess differences in gender composition among the 3 groups. The mean age of the three groups was also compared. 


\section{Results}

We identified a definite etiology for the sudden hearing loss (group DE) in 13 subjects with 14 involved ears (10\%). There were 6 cases of Ménière's disease, 3 cases of acoustic schwannoma, 1 case of temporal bone meningioma, 1 case of cochlear inflammatory disease, 1 case of mumps, and 1 case of Cogan syndrome that involved both ears. The case of cochlear auto-immune disease was identified by MRI and subsequently confirmed by measurement of HSP 70. The patient with mumps developed hearing loss during the course of the clinical disease and also exhibited inflammatory cochlear MRI findings. MRI also discovered the VIII schwannoma and the meningioma; the clinical evolution and audiometry identified the patients with Ménière's disease, and biochemical tests, clinical history and ophthalmologic examination identified the case of Cogan syndrome.

A concurrent disease (CD group) was identified in 63 subjects with 64 involved ears (46\%): hypertension (42 ears with one bilateral case), diabetes (18 ears), dyslipidemia (6 ears), upper airway infection with normal inner ear MRI (6 ears), arthritis (4 ears), arrhythmia (3 ears), thyroid disease (3 ears), chronic renal insufficiency (2 ears), coronary insufficiency (2 ears), systemic lupus erythematosus ( 1 ear), and convulsive crisis (1 ear). More than one coexistent disease was evident in some subjects and $73 \%$ of the patients from this group presented metabolic decompensation at the initial evaluation.

Sixty subjects with 61 involved ears $(43.9 \%)$ had no specific etiology or concurrent disease and comprised the ISSHL group. The presence of tinnitus was observed in $78 \%$ of DE cases, $87 \%$ of CD cases, and $87 \%$ of ISSHL cases. Vertigo or imbalance was detected in $56 \%$ of DE cases, $43 \%$ of CD cases and $39 \%$ of ISSHL cases.

There was no significant difference in age between the DE (41.93 years) and ISSHL (39.13 years) groups. However, the CD group was significantly older (53.80 years) than DE $(P=0.016)$ and ISSHL groups $(P=0.001)$. The Pearson $\chi^{2}$ test detected no difference in gender composition among the 3 groups ( $P=0.256)$.

In the DE group, the hearing loss at presentation was classified as mild in $21.4 \%$ of the 14 ears, moderate in $50 \%$, severe in $21.4 \%$, and profound in $7.1 \%$. None of the ears exhibited a total hearing loss in this group. For group $\mathrm{DE}$ as a whole, the mean auditory threshold at presentation was $59.6 \mathrm{~dB}$ and the final mean threshold was $56.9 \mathrm{~dB}$. The hearing loss was totally recovered in $28.6 \%$, partially recovered in $21.4 \%$, remained essentially unchanged in $21.4 \%$, and worsened in $28.6 \%$. These percentages were similar across the subjects, with no correlation with initial

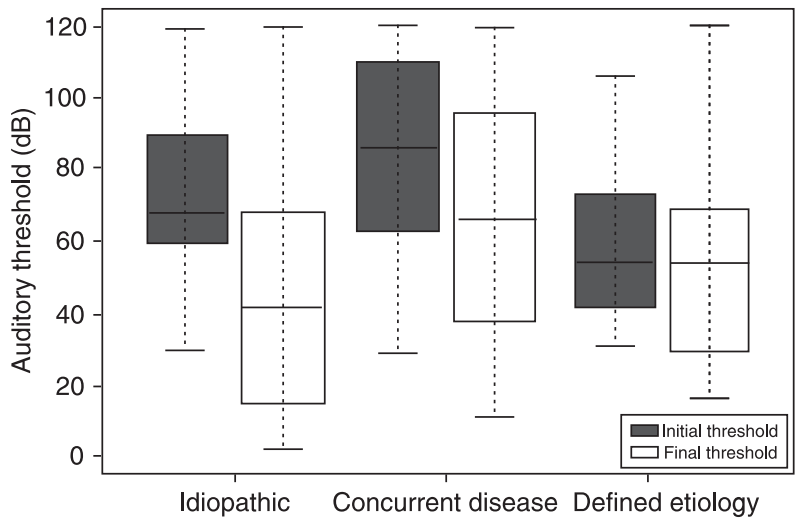

Figure 1. Mean initial and final auditory thresholds of the three groups.

hearing loss.

In the CD group (64 ears), $9.4 \%$ of the patients initially presented a mild hearing loss, $28.1 \%$ a moderate loss, $15.6 \%$ a severe loss, $31.3 \%$ a profound loss, and $15.6 \%$ had a total hearing loss in the involved ear. The initial and final mean auditory thresholds for the CD group as a whole were 82.7 and $66.3 \mathrm{~dB}$, respectively. In this group, $10.3 \%$ experienced total hearing recovery, $48.3 \%$ had partial recovery, $34.5 \%$ maintained the initial hearing levels, and $6.9 \%$ worsened.

The initial audiograms of the 61 ears in the ISSHL group showed mild hearing loss in $4.9 \%$, moderate in $47.5 \%$, severe in $23 \%$, profound in $16.4 \%$, and total in $8.2 \%$. Forty-four percent of these ears achieved total recovery of hearing at 6 months and $28 \%$ had partial improvement. There was no recovery in $22 \%$ and hearing worsened in $6 \%$. The initial mean auditory threshold in this group was $74.5 \mathrm{~dB}$ and the final mean threshold was 44.9 $\mathrm{dB}$.

Comparing the mean initial and final hearing loss for the three groups (marginal homogeneity test), a significant hearing improvement was observed for the $C D(P=0.001)$ and ISSHL $(P=0.001)$ groups. However, group DE did not show a statistically significant difference in hearing thresholds (Figure 1).

\section{Discussion}

Sudden sensorineural hearing loss is an intriguing clinical condition. It remains difficult to understand the outcomes of the natural history and the efficacy of different treatments for many reasons, including differences in standardization of its definition, different outcome measures studied, differences in patient demographics, and the existence of multiple factors involved in its genesis (9). In the 
present study, the degree of initial hearing loss was not evaluated as a prognostic item; the aim was to emphasize the etiology of cochlear aggression. Our protocol of investigation allowed us to identify a specific etiology in only $10 \%$ of cases. Nonetheless, the prognosis for hearing recovery was poorer in this group of patients with no significant hearing improvement, although some cases did improve using only the standard initial treatment, including two patients with vestibular schwannomas and one with a meningioma. The behavior of these patients was similar to that of the ISSHL group, emphasizing the importance of complete evaluation of all cases of sudden hearing loss $(10,11)$. The patients with Ménière disease and autoimmune disorders received specific therapy after the identification of these alterations. In the patient with Cogan syndrome, the hearing loss was the first symptom, again emphasizing the necessity of a complete investigation in patients with sudden hearing loss. Despite the effort to control the basic problem, no hearing improvement was observed in this group during a 6-month follow-up, suggesting that these specific diseases may promote chronic aggression to the inner ear and/or cochlear nerve, allowing limited hearing recovery.

Unfortunately, nearly $90 \%$ of our cases did not have an established etiology, but interestingly, when these patients were divided into subjects with concomitant disease and subjects without any other contributing factor, three significant findings were obtained: CD patients were older and had greater initial hearing impact and poorer hearing recovery at the conclusion of the study, although an important improvement occurred with the initial treatment regimen and associated therapy for the underlying disease initiated as soon as an affirmative diagnosis was possible (Figure 1).

Although assigning specific mechanisms for the hearing loss of these patients is only speculative, diseases such as diabetes, hypertension, dyslipidemia, and viral infection might have important effects on the physiopathology of hearing involvement through microvascular disturbances and cochlear inflammatory processes (12-14). The possibility of cochlear vascular involvement in these cases justified the use of pentoxifylline in our treatment regimen, but it is impossible to conclude from our study if the medication played an important role in the hearing recovery.

Although all CD patients with diabetes, dyslipidemia and hypertension were aware of their diseases, most of them presented clear decompensated status during the initial evaluation, representing evidence of inadequate treatment maintenance. The control of concurrent diseases provided an improvement of hearing level, even though not as significant as observed in patients without metabolic and vascular disorders. The occurrence of sudden hearing loss in these patients was important by calling attention to a more rigid control of those primary diseases.

The importance of age and the incidence of diabetes and hypertension in patients of the present study deserve some comments. According to the Brazilian Endocrinology Society, diabetes is found in $7.6 \%$ of the Brazilian general population between 30 and 69 years of age, but reaches $12.7 \%$ between ages 50 and 59 . The CD group presented a mean age of 53.8 years and $12.9 \%$ of these patients had diabetes. In this same group, $30.2 \%$ had associated hypertension, which affects $20 \%$ of the Brazilian general population and up to $47.8 \%$ of Brazilians between ages 50 and 59 . These data suggest that, although an expressive number of diabetic and hypertensive patients were detected in the CD group, the frequency of these diseases in SSNHL is comparable to that for the general Brazilian population of similar age. In conclusion, these vascular and metabolic disorders may contribute to the onset of, and can be a risk factor for, SSNHL but further prospective studies are needed to confirm the true effects of these metabolic diseases on the evolution of hearing loss.

The occurrence of decompensated metabolic and cardiovascular diseases and the possibility of first manifestation in autoimmune disease and in cerebello-pontine angle tumors justify an adequate protocol of investigation in patients with SSNHL.

In 61 instances, the cause of SSNHL remained unknown. We presume that the diagnosis of SSNHL will improve in the future with new and better testing (15). At present, a battery of studies similar to those described here provide a quick way to identify both the specific causes and concurrent diseases, which require specific medical intervention.

\section{Acknowledgments}

We thank the statistician Fábio Tadeu Montesano from Federal University of São Paulo for statistical analysis. 


\section{References}

1. National Institute of Health. Sudden deafness. Bethesda: National Institutes of Health, NIH publication No. 00-4757; 2000.

2. Wilson WR, Byl FM, Laird N. The efficacy of steroids in the treatment of idiopathic sudden hearing loss. A double-blind clinical study. Arch Otolaryngol 1980; 106: 772-776.

3. De Kleyn A. Sudden complete or partial loss of function of the octavus system in apparently normal persons. Acta Otolaryngol 1944; 32: 407-429.

4. Penido NO, Ramos HVL, Barros FA, Cruz OLM, Toledo R. Clinical and etiological factors and evolution of hearing in sudden deafness. Rev Bras Otorrinolaringol 2005; 71: 633 638.

5. Ramos HVL, Yamashita H, Barros FA, Souza ACV, Yamaoka WY, Penido NO. Magnetic resonance imaging in sudden deafness. Rev Bras Otorrinolaringol 2005; 71: 422426.

6. Weng SF, Chen YS, Liu TC, Hsu CJ, Tseng FY. Prognostic factors of sudden sensorineural hearing loss in diabetic patients. Diabetes Care 2004; 27: 2560-2561.

7. Committee on Hearing and Equilibrium guidelines for the diagnosis and evaluation of therapy in Meniere's disease. American Academy of Otolaryngology - Head and Neck Surgery Foundation, Inc. Otolaryngol Head Neck Surg 1995; 113: 181-185.

8. Shiraishi T, Kubo T, Okumura S, Naramura H, Nishimura M, Okusa $\mathrm{M}$, et al. Hearing recovery in sudden deafness patients using a modified defibrinogenation therapy. Acta Oto- laryngol Suppl 1993; 501: 46-50.

9. Ziegler EA, Hohlweg-Majert B, Maurer J, Mann WJ. [Epidemiological data of patients with sudden hearing loss - a retrospective study over a period of three years]. Laryngorhinootologie 2003; 82: 4-8.

10. Sauvaget E, Kici S, Kania R, Herman P, Tran Ba HP. Sudden sensorineural hearing loss as a revealing symptom of vestibular schwannoma. Acta Otolaryngol 2005; 125: 592-595.

11. Nageris BI, Popovtzer A. Acoustic neuroma in patients with completely resolved sudden hearing loss. Ann Otol Rhinol Laryngol 2003; 112: 395-397.

12. Hirano K, Ikeda K, Kawase $T$, Oshima $T$, Kekehata $S$, Takahashi S, et al. Prognosis of sudden deafness with special reference to risk factors of microvascular pathology. Auris Nasus Larynx 1999; 26: 111-115.

13. Weng SF, Chen YS, Hsu CJ, Tseng FY. Clinical features of sudden sensorineural hearing loss in diabetic patients. Laryngoscope 2005; 115: 1676-1680.

14. Fukui M, Kitagawa $\mathrm{Y}$, Nakamura N, Kadono M, Mogami S, Ohnishi $\mathrm{M}$, et al. Idiopathic sudden hearing loss in patients with type 2 diabetes. Diabetes Res Clin Pract 2004; 63: 205211.

15. Cadoni G, Agostino S, Scipione S, Ippolito S, Caselli A, Marchese R, et al. Sudden sensorineural hearing loss: our experience in diagnosis, treatment, and outcome. J Otolaryngol 2005; 34: 395-401. 